\title{
Parámetros de Transferencia de Materia en el Secado de Frutas, sin Necesidad de Datos de Disminución de Volumen
}

\author{
Sebastião R. Ferreira y Antônio R. S. Costa \\ Universidad Federal de Río Grande del Norte, Departamento de Ingeniería Química, \\ Av. Salgado Filho 3.000, 59078-970 Natal, RN-Brasil (e-mail: ferreira@eq.ufrn.br, seba@ufrnet.br)
}

Recibido Sept. 11, 2009; Aceptado Nov. 20, 2009; Versión Final recibida Dic. 29, 2009

\begin{abstract}
Resumen
Se desarrolló una metodología analítica para evaluar parámetros de transferencia de materia en el secado de frutas cilíndricas, sin necesidad de conocer datos de disminución de volumen, pero admitiendo implicitamente disminución de su radio en el proceso R. Con el procedimiento se predice el coeficiente externo de transferencia de materia $k_{m}$, la difusividad efectiva del agua en frutos $D_{\text {ef }}$, el número de Biot $\mathrm{Bi}$ y el radio promedio $\mathrm{R}_{\mathrm{m}}$ durante el secado. Para probar la metodología es necesario obtener datos de masa de agua en una fruta $M_{A}$ versus tiempo $t$, y sus dimensiones características en el comienzo del proceso. Los valores obtenidos de $\mathrm{Bi}, \mathrm{D}_{\text {ef }}$ y $\mathrm{k}_{\mathrm{m}}$ se ajustan bien a otros encontrados en la literatura, calculados con un método numérico. La metodología desarrollada puede ser empleada para evaluar parámetros de secado con buena exactitud, pero sin necesidad de obtener datos experimentales de disminución de volumen.
\end{abstract}

Palabras clave: parámetros de transferencia de materia, difusividad efectiva del agua, transferencia de materia, secado de frutas, modelado matemático.

\section{Mass Transfer Parameters in Fruit Drying, without using Shrinkage Data}

\begin{abstract}
An analytical methodology was developed to determine mass transfer parameters during cylindrical fruit drying, without the need for shrinkage data, but implicitly considering radius shrinkage $\mathrm{R}$ during the drying process. With the method one can simultaneously evaluate the external mass transfer coefficient $k_{m}$, the effective water diffusivity in fruit $D_{e f}$, the Biot number $B i$ and the average radius $R_{m}$ during drying. To test the methodology, experimental data of the water mass in fruit $M_{A}$ versus time $t$, and its characteristic dimensions at the start of process were needed. The values obtained for $\mathrm{Bi}$, Def and $\mathrm{km}$ were in good agreement with values found in the literature, calculated by a numerical method. The proposed methodology can be used to estimate drying parameters with good accuracy without the need of shrinkage data.
\end{abstract}

Keywords: mass transfer parameters, effectively water diffusivity, mass transfer, fruit drying, mathematical modeling. 


\section{INTRODUCCIÓN}

A partir de modelos matemáticos de secado de frutas surgen naturalmente parámetros que influyen en el proceso, tales como el coeficiente externo de transferencia de materia $\mathrm{k}_{\mathrm{m}}(\mathrm{m} / \mathrm{s})$, la difusividad efectiva del agua en una fruta $\mathrm{D}_{\text {ef }}\left(\mathrm{m}^{2} / \mathrm{s}\right)$ y el número de Biot $\mathrm{Bi}($ adim.). Uno de los más conocidos modelos de secado es el de Luikov (1966), que está basado en la termodinámica de los procesos irreversibles (Abalone et al., 2001; Luikov, 1966; Pandey et al., 1999; Wu e Irudayaraj, 1996).

En algunas investigaciones están contemplados modelos de secado de frutos (Lima, 1999) así como de secador (Karim y Hawlader, 2005a, b; Mabrouk et al., 2006). Los modelos para el fruto y secador están acoplados a través de flujos de materia, energía y cantidad de movimiento en la interfase fruta/aire. Lima (1999) analizó el secado de banana y reprodujo parte de su investigación en un artículo (Lima et al., 2002). En su trabajo original (Lima, 1999) desarrolló modelos bidimensionales, analíticos y numéricos, para simular la difusión de agua en sólidos, los cuales fueron empleados para describir el secado de banana. En su investigación se destaca el denominado modelo (III), en el cual usa condición de contorno convectiva, fenómenos simultáneos de transferencia de humedad y disminución de volumen del fruto. En su modelo (III) el citado autor admitió, entre otras consideraciones, que: a) El secado ocurre bajo condición de contorno convectiva, con humedad dependiente de la posición y del tiempo, en un sólido esferoidal; del cual se presenta un esquema en la Fig. 1; b) El número de Biot es admitido como variable en el secado; el cual es definido por la Ec.(3); y c) La disminución de volumen es linealmente proporcional a la pérdida promedio de humedad del sólido.

Según Lima, el modelo (III) es el más realista de los analizados por él, dando resultados confiables para los parámetros transferencia de materia; porque están incluidos más efectos físicos que inciden en la cinética de secado, comparado con otros modelos de citado autor (Lima, 1999; Lima et al., 2002). En la Fig.1, que representa la mitad de la longitud de una banana, están destacados los sistemas de coordenadas cartesianas $(x, y, z)$ y esferoidal $(\mu, \phi, \omega)$. Se presentan el semieje menor y mayor, $L_{1}$ y $L_{2}$, respectivamente, y la longitud focal L, que es calculada por la ecuación (Lima, 1999):

$$
L=\sqrt{\left(L_{2}^{2}-L_{1}^{2}\right)}
$$

El volumen de un sólido esferoidal, con $L_{1}<L_{2}$, es calculado por la ecuación (Lima, 1999):

$$
V=\frac{4}{3} \pi L_{1}^{2} L_{2}
$$

El número de Biot de transferencia de materia $\mathrm{Bi}_{\mathrm{L}}$, para un sólido esferoidal de longitud característica $\mathrm{L}$, como el de la Fig.1, es dado por (Lima, 1999):

$$
B i_{L}=k_{m} L / D_{\text {ef }}
$$

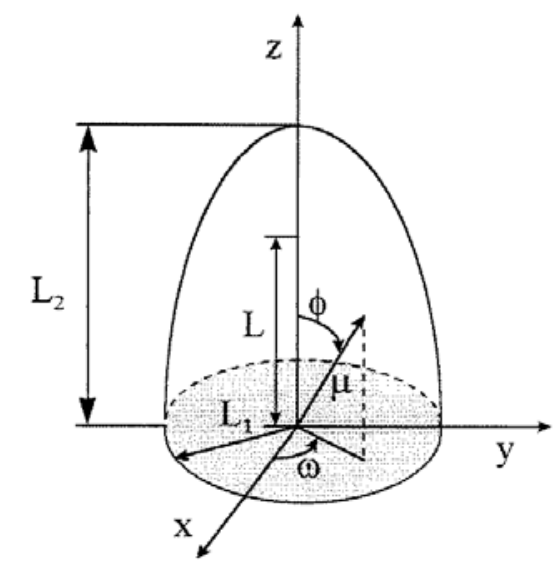

Fig. 1: Características de un sólido esferoidal (Lima, 1999; Teruel et al., 2001). 
Una banana es un sólido esferoidal, pero si son cortadas sus dos puntas, se obtiene aproximadamente un cilindro finito $y$, en general, el valor de $L_{1}$ es casi igual al radio de una banana $R$, y $L$ es casi igual a la mitad de su longitud. Para una banana, admitida como cilindro finito, existen dos números de Biot de transferencia de materia; uno para la longitud $\mathrm{L}, \mathrm{Bi}_{\mathrm{L}}$, evaluado con la $\mathrm{Ec}$.(3) y otro para el radio $\mathrm{R}$ o la longitud $L_{1} \approx R, B i_{R}$, calculado por la Ec.(6). Para un cilindro infinito sólo hay Biot para $R \circ L_{1}$. Para comparar un modelo de transferencia de materia de cilindro infinito con un modelo de sólido esferoidal, hay que dividir sus números de Biot, $\mathrm{Bi}_{R}$ y $\mathrm{Bi}_{\mathrm{L}}$, incluyendo sus dimensiones $\mathrm{R}$ y $\mathrm{L}$, obteniendo así el orden de magnitud de cada Bi. Lo mismo debe ser realizado para los coeficientes $\mathrm{k}_{\mathrm{m}}$. Para relacionar modelos de transferencia de materia de cilindro infinito, uno analítico con otro numérico, se puede comparar directamente el $\mathrm{Bi}$ radial $\mathrm{Bi}_{\mathrm{R}}$ de un modelo con el $\mathrm{Bi}_{\mathrm{R}}$ de otro modelo; lo que simplifica el análisis en relación a comparar con sólido esferoidal. Similares comparaciones pueden ser realizadas entre un modelo de cilindro finito con uno infinito o entonces uno de cilindro finito con uno de sólido esferoidal etc.

Karim y Hawlader (2005a, b) desarrollaron un modelo de secado de frutas, prediciendo su temperatura y humedad. Como ellos admitieron disminución de volumen, la difusividad $\mathrm{D}_{\text {ef }}$ resultó dependiente del contenido acuoso y de la temperatura (Bird et al., 2002; Crank, 1976), y disminuyó durante el secado. Mabrouk et al. (2006) emplearon un modelo numérico para predicción de la transferencia de materia y energía de productos granulares en un secador tipo túnel. Como la capa usada de producto (uva) era de pequeño espesor, ellos admitieron que no existía gradiente de temperatura y humedad, y obtuvieron un modelo simplificado basado en una capa delgada. Costa (2008), desarrolló un modelo de secado de un cilindro infinito, basándose en la ecuación de Fick. Ferreira y Costa (2009), presentaron un modelo de secado de banana, admitiendo disminución de radio del fruto y emplearon datos de disminución de volumen de la literatura (Lima, 1999; Queiroz, 1994), para probar su modelo. Ferreira y Costa (2009) evaluaron $\mathrm{Bi}_{\mathrm{m}}, \mathrm{D}_{\text {ef }} \mathrm{y} \mathrm{k}_{\mathrm{m}}$, empleando un programa de regresión no lineal, usando datos de masa de agua y radio de una fruta versus tiempo. Concluyeron que los parámetros calculados eran del mismo orden de magnitud que los evaluados por Lima (1999).

Una de las dificultades encontradas en el modelado de secado es la obtención de parámetros de transferencia de materia y energía, tales como el coeficiente convectivo de transferencia de calor, $\mathrm{h}_{\text {conv }}\left(\mathrm{Wm}^{-20} \mathrm{C}^{-1}\right), \mathrm{Bi}_{\mathrm{m}}, \mathrm{D}_{\text {ef }} \mathrm{y} \mathrm{k}_{\mathrm{m}}$, que representen bien el fenómeno. Es común emplear correlaciones $\mathrm{o}$ experimentos por separado para calcularlos o usar métodos numéricos, para analizar como ellos se comportan en función de variables del proceso. Para resolver simultáneamente las ecuaciones de transferencia de materia y energía, Mariani et al. (2008) emplearon correlaciones para $\mathrm{h}_{\text {conv, }}$ en vez de calcularlo automáticamente del método numérico usado. En general, esas correlaciones sólo son válidas para una geometría dada, en función de los números de Nusselt, Prandtl y Reynolds (Bird et al., 2002).

El $h_{\text {conv }}$ puede ser evaluado de ensayo por separado, por ejemplo, con un cilindro de aluminio, de iguales dimensiones y en condiciones similares que una fruta dada, resultando para el cilindro metálico que $\mathrm{Bi}_{\mathrm{C}}=$ $h_{\text {conv }} R / k_{A l} \rightarrow 0$. Como la conductividad térmica del aluminio $k_{A l}$ es muy grande, en general, se cumple que $\mathrm{Bi}_{\mathrm{C}} \rightarrow 0 \mathrm{y} \mathrm{h}_{\text {conv }}$ es calculado de la curva de $\mathrm{T}$ versus $\mathrm{t}$, en el cilindro de aluminio, con un modelo sencillo de transferencia de calor. El $\mathrm{h}_{\text {conv }}$ obtenido para el cilindro metálico puede ser usado para una fruta, en las condiciones citadas. Srikiatden y Roberts (2008) evaluaron $h_{\text {conv }}$, con un procedimiento similar al citado, para calcular $D_{\text {ef }}$ de agua en papas y zanahorias. Kaya et al. (2008) obtuvieron el coeficiente local $\mathrm{h}_{\text {conv }}$ de experimentos, con flujo de calor en la superficie de kiwi y calcularon $\mathrm{k}_{\mathrm{m}}$, con la analogía de Chilton y Colburn; entre el espesor de capa límite de transferencia de calor y materia. Lima (1999) evaluó $\mathrm{k}_{\mathrm{m}}$ en el secado de banana, ajustando un modelo matemático, usando datos de Queiroz (1994).

En el presente trabajo se propone una metodología para evaluar parámetros tales como $B i, D_{e f}, k_{m} y R_{m}$, sin necesidad de conocer datos de cambio del radio de una fruta cilíndrica en su secado, pero sólo conociendo principalmente sus dimensiones iniciales y datos de $M_{A}$ versus t. Los parámetros $B i, D_{\text {ef }}, R_{m}$, $M_{A 0}$ y $M_{A \infty}$ son mantenidos libres en los cálculos, siendo los dos últimos, respectivamente, la masa de agua en una fruta en el inicio del secado y a tiempo infinito. Los parámetros calculados con el método analítico de cilindro infinito, son comparados con los de otra metodología analítica de cilindro infinito, pero usando datos de disminución de volumen (Ferreira y Costa, 2009), y con un método numérico, empleando datos de disminución de volumen, de un sólido esferoidal (Lima, 1999). 


\section{MODELO PARA SECADO DE UN CILINDRO INFINITO}

El modelo de secado empleado en el presente artículo ya fue discutido en la literatura (Ferreira y Costa, 2009). Los citados autores analizaron la relación entre la difusividad de agua en una fruta $D_{\text {ef }}\left(\mathrm{m}^{2} / \mathrm{s}\right)$ y la difusividad térmica $\alpha\left(\mathrm{m}^{2} / \mathrm{s}\right)$, dada por el número de Luikov $L u=(1 / \alpha) /\left(1 / D_{\mathrm{ef}}\right)=($ resistencia interna a la difusión de calor)/(resistencia interna a la difusión de materia). Por ejemplo, para secado en aire a 29,9 ${ }^{\circ} \mathrm{C}$, de banana de conductividad térmica $\mathrm{k}=0,481 \mathrm{Wm}^{-10} \mathrm{C}^{-1}$, densidad $\rho=980 \mathrm{~kg} / \mathrm{m}^{3}$, calor específico $\mathrm{Cp}$ $=3.346 \mathrm{Jkg}^{-10} \mathrm{C}^{-1}$ y $D_{\text {ef }}=1,63 \cdot 10^{-10} \mathrm{~m}^{2} / \mathrm{s}$, se calcula que $\mathrm{Lu}=\left(D_{\mathrm{ef}} \rho \mathrm{Cp}\right) / \mathrm{k}=0,00111<<1$. Como se cumple que $\mathrm{Lu} \ll<1$, la transferencia interna de materia domina las transferencias simultáneas de calor y materia. Con las consideraciones realizadas, la ecuación de difusión de Fick puede ser empleada como la ecuación diferencial básica para un análisis simplificado.

La ecuación de Fick para difusión radial en una fruta, admitida como siendo un cilindro infinito, así como las condiciones de contorno, las cuales son condición de contorno convectiva, condición de simetría radial y masa inicial de agua $\mathrm{M}_{\mathrm{AO}}(\mathrm{g})$, fueron presentadas y discutidas en otro trabajo (Ferreira y Costa, 2009). La solución de la ecuación de Fick, que da la masa de agua en una fruta $M_{A}(t)$, con las condiciones de contorno mencionadas, está dada por las ecuaciones a continuación (Crank, 1976; Luikov, 1968):

$$
\begin{aligned}
& \frac{M_{A}-M_{A \infty}}{M_{A 0}-M_{A \infty}}=\sum_{n=1}^{\infty} B_{n} \exp \left(-\mu_{n}^{2} D_{e f} t / R^{2}\right) \\
& B_{n}=\frac{4 B_{R}^{2}}{\mu_{n}^{2}\left(\mu_{n}^{2}+B i_{R}^{2}\right)} \\
& B_{R}=k_{m} R / D_{e f}
\end{aligned}
$$

Siendo $B_{n}$ un parámetro, $t(s)$ el tiempo y $\mu_{n}($ adim.) los autovalores, para condición de contorno convectiva. Con la Ec.(4) se calcula $\mathrm{M}_{\mathrm{A}}$ versus $\mathrm{t}$, en función de Biot en el radio de un cilindro infinito $\mathrm{Bi}_{\mathrm{R}}$, de la Ec.(6). De la Ec.(4) también se pueden evaluar $B i_{R}, D_{e f}, M_{A 0}, M_{A \infty}$ y un radio promedio $R_{m}$, si fueren conocidos $M_{A}$ versus t. Para realizar cálculos con la Ec.(4), son necesarias las ecuaciones presentadas a continuación:

$$
\begin{aligned}
& \operatorname{Bi}_{R} J_{0}\left(\mu_{n}\right)=\mu_{n} J_{1}\left(\mu_{n}\right) \\
& J_{0}\left(\mu_{n}\right)=1-\frac{\mu_{n}^{2}}{2^{2}}+\frac{\mu_{n}^{4}}{2^{2} 4^{2}}-\frac{\mu_{n}^{6}}{2^{2} 4^{2} 6^{2}}+\ldots \\
& J_{1}\left(\mu_{n}\right)=\frac{\mu_{n}}{2}-\frac{\mu_{n}^{3}}{2^{2} 4}+\frac{\mu_{n}^{5}}{2^{2} 4^{2} 6}-\frac{\mu_{n}^{7}}{2^{2} 4^{2} 6^{2} 8}+\ldots
\end{aligned}
$$

La Ec.(7) es la ecuación de autovalores $\mu_{n}$, la cual ayuda en la obtención de $M_{A}$ de la Ec.(4). Las Ecs.(8) y (9) son, respectivamente, la función de Bessel de primera especie, de orden cero $\mathrm{J}_{0}\left(\mu_{n}\right)$ y uno $\mathrm{J}_{1}\left(\mu_{n}\right)$.

\section{CÁLCULO DE PARÁMETROS DEL MODELO DE SECADO}

Es presentada una metodología para evaluar parámetros, tales como $B_{R}, D_{e f}, M_{A 0}, M_{A \infty}$ y $R_{m}$, basándose en la Ec.(4); y enseguida $\mathrm{Bi}_{\llcorner}$y $\mathrm{k}_{\mathrm{m}}$. Para realizar cálculos con la Ec.(4) con sólo el primer autovalor $\mu_{1}$, es mejor emplear una correlación en vez de resolver la Ec.(7). Para cumplir con tal objetivo, son usadas las ecuaciones a continuación, de $\mu_{1}$ versus Bi, presentadas por Schwartzberg (1981), para cilindro infinito:

$$
\mu_{1}=\sqrt{2 \mathrm{Bi} /(1+0,26 \mathrm{Bi})} \text { Para } \mathrm{Bi}<2
$$




$$
\mu_{1}=\frac{2,4048 \mathrm{Bi}}{1,03+\mathrm{Bi}} \text { Para } \mathrm{Bi}>2
$$

El rango de Biot presentado por Schwartzberg (1981) para las Ecs.(10) y (11) es, respectivamente, $\mathrm{Bi}<3$ y $\mathrm{Bi}>5$; no contemplando $3<\mathrm{Bi}<5$. Las Ecs.(10) y (11) fueron evaluadas por los autores del presente artículo, que encontraron que, en general, ellas dan desviaciones menores que $\mid 1,5 \%$ en relación a la Ec.(7), respectivamente, para $\mathrm{Bi}<2$ y $\mathrm{Bi}>2$. Los cálculos con el modelo de la sección anterior se simplifican mucho, en parte, por ser empleada una correlación como la Ec.(10) o (11). Las masas $\mathrm{M}_{\mathrm{Ao}} \mathrm{y}$ $\mathrm{M}_{\mathrm{A}}$ son parámetros libres en el presente trabajo, pero no lo fueron en el de Ferreira y Costa (2009). Éste artificio elimina la necesidad de conocer datos de disminución de volumen, mejorando la precisión de cálculo de parámetros; cuando se usa sólo el primer término de la Ec.(4). Los valores de $M_{A 0}$ y $M_{A \infty}$ pueden diferir de los experimentales o calculados por correlaciones, pues son parámetros de ajuste.

El procedimiento para evaluar los parámetros $B i_{R}, D_{e f}, M_{A 0}, M_{A \infty}, R_{m}, B i_{L} y k_{m}=k_{L}$, es:

Son obtenidos datos experimentales de $M_{A}$ versus $t$, el radio de una fruta al comienzo del proceso $R_{0} \approx$ $L_{10}$ y su semieje mayor $L_{20}$.

Es reemplazado $\mu_{1}$ en el primer término de la Ec.(4), por ejemplo, $\mu_{1}$ de la Ec.(11), si $\mathrm{Bi}_{\mathrm{R}}>2$. La sustitución es realizada tanto en el factor preexponencial de la Ec.(4), dado por B ${ }_{1}$ de la Ec.(5), así como dentro del exponencial, reemplazando el autovalor al cuadrado, es decir, elevando la Ec.(11) al cuadrado, $\mu_{1}{ }^{2}$. Después de las citadas sustituciones, resulta del primer término de la Ec.(4):

$$
\frac{M_{A}-M_{A \infty}}{M_{A 0}-M_{A \infty}}=\frac{4\left(1,03+B i_{R}\right)^{4}}{\left(2,4048 B i_{R}\right)^{2}\left\{2,4048^{2}+\left(1,03+B i_{R}\right)^{2}\right\}} \exp \left\{-\left(\frac{2,4048 B i_{R}}{1,03+B i_{R}}\right)^{2} \frac{D_{e f} t}{R_{m}^{2}}\right\}
$$

Son correlacionados $M_{A}$ versus t, de la Ec.(12), con un programa de regresión no lineal, obteniendo $B i_{R}$, $D_{\text {ef }}, M_{A 0}, M_{A \infty}$ y $R_{m}$. $R_{m}$ es un promedio para el secado y es obtenido naturalmente del programa.

Es evaluada la longitud focal en el inicio del proceso de secado $L_{0}$, empleando datos de $L_{10}$ y $L_{20}$, el semieje menor y mayor, respectivamente, con la Ec.(1):

$$
L_{0}=\sqrt{\left(L_{20}^{2}-L_{10}^{2}\right)}
$$

Se considera que, en el comienzo del proceso, el semieje menor es casi igual a $L_{10} \approx R_{0}$ y que el representativo de la etapa final de secado es $L_{1 f} \approx R_{m}$; siendo $R_{m}$ obtenido de la Ec.(12). Se hacen las simplificaciones citadas porque no se emplea una ecuación de $R$ versus $M_{A}$, para evaluar el cambio de $R$ en el proceso. Se evalúa el semieje mayor del final del secado $L_{2 f}$, por:

$$
L_{2 f} \cong L_{0} R_{m} / R_{0} \cong L_{0} L_{1 f} / L_{10}
$$

De la Fig.1 se obtiene $L_{2 f}=\left(L_{20} / L_{10}\right) L_{1 f}$, que difiere de $L_{2 f}$ de la Ec.(14); pero la Ec.(14) fue elegida para cálculo porque se obtiene mejor ajuste empleándola en la presente investigación, especialmente para $\mathrm{Bi}$ al final del proceso $B i_{L f}$, en vez de usar $L_{2 f}=\left(L_{20} / L_{10}\right) L_{1 f}$.

Se evalúa la longitud focal $L_{f}$ al final del secado, con $L_{1 f} \approx R_{m}$ obtenido de correlación de la Ec.(12) y $L_{2 f}$ de la Ec.(14), resultando:

$$
L_{f} \cong \sqrt{\left(L_{2 f}^{2}-L_{1 f}^{2}\right)} \cong \sqrt{\left(L_{2 f}^{2}-R_{m}^{2}\right)}
$$


Es dividido el Biot de cilindro infinito $B i_{R}=k_{m} R_{m} / D_{\text {ef }}$ de la Ec.(6), por el de sólido esferoidal $B i_{L}=k_{m} L / D_{\text {ef }}$ de la Ec.(3), obteniendo $B i_{L}$ de un sólido esferoidal en el inicio $B i_{L 0}$ y final del proceso $B i_{L f}$, dados por las ecuaciones a continuación, con $L_{0}$ y $L_{f}$ de las Ecs.(13) y (15), respectivamente, y $B i_{R}$ y $R_{m}$ de la Ec.(12):

$$
\begin{aligned}
& B i_{L 0}=B i_{R} L_{0} / R_{m} \\
& B i_{L f}=B i_{R} L_{f} / R_{m}
\end{aligned}
$$

Es calculado el coeficiente externo de transferencia de materia de la Ec.(3), en el inicio $k_{m 0}=k_{L 0}$ y final del secado $k_{m f}=k_{L f}$, de un sólido esferoidal, con $B i_{L 0}$ y $B i_{L f}$, de las Ecs.(16) y (17), respectivamente, $L_{0}$ y $L_{f}$ de las Ecs.(13) y (15), respectivamente, y $D_{\text {ef }}$ de la Ec.(12):

$$
\begin{aligned}
& \mathrm{k}_{\mathrm{L} 0}=\mathrm{Bi}_{\mathrm{L} 0} \mathrm{D}_{\text {ef }} / \mathrm{L}_{0} \\
& \mathrm{k}_{\mathrm{Lf}}=\mathrm{Bi}_{\mathrm{Lf}} \mathrm{D}_{\text {ef }} / \mathrm{L}_{\mathrm{f}}
\end{aligned}
$$

Muchos experimentos de secado son conducidos a tiempos largos, resultando en grandes valores del número de Fourier promedio, $\mathrm{Fo}_{\mathrm{m}}=\mathrm{D}_{\mathrm{ef}} \mathrm{t}_{\mathrm{m}} / \mathrm{R}_{\mathrm{m}}{ }^{2}$, en general $\mathrm{Fo}_{\mathrm{m}}>0,5$, con lo que es suficiente usar sólo el primer término de la Ec.(4). Para mejorar el ajuste de parámetros, se puede aumentar la cantidad de términos empleados de la Ec.(4), usando $\mu_{1}$ en el primer término para calcular $B i_{R}$ y $D_{\text {ef }}$, y en los otros términos incluyendo parámetros $\mathrm{F}, \mathrm{G}, \mathrm{H}$ e l, tal como es presentado en la ecuación a continuación, para $\mathrm{Bi}_{\mathrm{R}}>2$. En éste artículo no fue realizado lo citado con anterioridad, pues con el programa de regresión empleado sólo es posible escribir ecuaciones con hasta 94 cifras; por ejemplo, 2,4048 fue escrito como 2,4. Pero, Lima (1999) correlacionó datos, de $M_{A}$ versus t, con una serie de tres términos.

$$
\frac{M_{A}-M_{A \infty}}{M_{A 0}-M_{A \infty}}=\frac{4\left(1,03+B i_{R}\right)^{4}}{\left(2,4048 B i_{R}\right)^{2}\left\{2,4048^{2}+\left(1,03+B i_{R}\right)^{2}\right\}} \exp \left\{-\left(\frac{2,4048 B i_{R}}{1,03+B i_{R}}\right)^{2} \frac{D_{\text {ef }} t}{R_{m}^{2}}\right\}+F \exp (-G t)+H \exp (-I t)
$$

Se puede obtener una ecuación de cálculo de $\mathrm{D}_{\text {ef }}$ para la situación límite de $\mathrm{Bi} \rightarrow \infty$; que usando el primer término de la Ec.(4) o de la Ec(12), resulta en la ecuación presentada a continuación, después de evaluar $\mu_{1}=2,4048$ de la Ec.(7) o (11) y el parámetro $B_{1}=4 /(2,4048)^{2}=0,6917$ de la Ec.(5):

$$
\frac{M_{A}-M_{A \infty}}{M_{A 0}-M_{A \infty}}=\frac{4}{(2,4048)^{2}} \exp \left\{-(2,4048)^{2} D_{e f} t / R_{m}^{2}\right\}
$$

La Ec.(21) es un límite, por ejemplo, para el cálculo de la difusividad $\mathrm{D}_{\text {ef }} \mathrm{y}$ sólo es válida cuando $\mathrm{Bi} \rightarrow \infty$. La Ec.(21) debe ser usada con cautela y solamente cuando no hay resistencia externa a la transferencia de materia. Por lo tanto, los resultados obtenidos de la Ec.(21) sólo se acercan a la realidad de un proceso de secado, si $\mathrm{Bi} \rightarrow \infty$ y para un Fourier promedio de aproximadamente $\mathrm{Fo}_{\mathrm{m}}=\mathrm{D}_{\mathrm{ef}} \mathrm{t}_{\mathrm{m}} / \mathrm{R}_{\mathrm{m}}{ }^{2}>0,5$.

Para relacionar la metodología analítica propuesta de cilindro infinito, con un método numérico de cilindro infinito, se compara directamente el $\mathrm{Bi}$ radial $\mathrm{Bi}_{R}$ de un modelo con el $\mathrm{Bi}_{R}$ del otro. Es decir, el $\mathrm{Bi}_{R}$ calculado por regresión de la Ec.(12) es el de la etapa final, $B_{R}=B_{R f}$. El del comienzo es evaluado con el radio inicial $R_{0}, B i_{R 0}=B i_{R f} R_{0} / R_{m}$. $k_{m}=k_{m f}$ es calculado con $B i_{R f}, D_{\text {ef }} y R_{m}$, resultando en $k_{m f}=B i_{R f} D_{e f} / R_{m}$, así como $k_{m 0}=B i_{R 0} D_{e f} / R_{0}$. Si es empleado éste procedimiento, muchas de las ecuaciones discutidas previamente no son necesarias para los cálculos.

\section{RESULTADOS Y DISCUSIÓN}

Son realizados cálculos con la metodología propuesta, usando datos de Queiroz (1994), Lima (1999) y Lima et al. (2002); algunos de los cuales están reproducidos en la Tabla 1. 
Tabla 1: Parámetros del aire y trozo de banana de ensayos de secado (Lima, 1999; Queiroz, 1994).

\begin{tabular}{|c|c|c|c|c|c|c|c|c|c|c|c|}
\hline \multirow[t]{2}{*}{ Ensayo } & \multicolumn{3}{|c|}{ Datos del aire } & \multicolumn{7}{|c|}{ Datos de un trozo de banana } & \multirow{2}{*}{$\begin{array}{c}\text { Tiempo } \\
\text { de } \\
\text { secado } \\
\text { (h) }\end{array}$} \\
\hline & $\begin{array}{c}\mathrm{T}_{\mathrm{a}} \\
\left({ }^{\circ} \mathrm{C}\right)\end{array}$ & $\begin{array}{l}\text { UR } \\
(\%)\end{array}$ & $\begin{array}{c}\mathrm{v} \\
(\mathrm{m} / \mathrm{s})\end{array}$ & $\begin{array}{c}\mathrm{U}_{0} \\
\text { (b.s) }\end{array}$ & $\begin{array}{c}U_{f} \\
\text { (b.s) }\end{array}$ & $\begin{array}{c}\mathrm{U}_{\mathrm{e}} \\
\text { (b.s) }\end{array}$ & $\begin{array}{c}\mathrm{T}_{0} \\
\left({ }^{\circ} \mathrm{C}\right)\end{array}$ & $\begin{array}{c}\mathrm{T}_{\mathrm{f}} \\
\left({ }^{\circ} \mathrm{C}\right)\end{array}$ & $\begin{array}{l}\mathrm{L}_{10} \\
(\mathrm{~m})\end{array}$ & $\begin{array}{l}\mathrm{L}_{20} \\
(\mathrm{~m})\end{array}$ & \\
\hline 1 & 29,9 & 35,7 & 0,38 & 3,43 & 0,32 & 0,1428 & 19,1 & 29,7 & 0,01613 & 0,05856 & 121,85 \\
\hline 2 & 39,9 & 19,3 & 0,33 & 3,17 & 0,33 & 0,0664 & 21,0 & 38,9 & 0,01569 & 0,05878 & 72,00 \\
\hline 3 & 49,9 & 19,2 & 0,37 & 3,21 & 0,32 & 0,0579 & 20,3 & 47,1 & 0,01522 & 0,05901 & 40,80 \\
\hline 4 & 60,2 & 19,9 & 0,36 & 2,96 & 0,25 & 0,0426 & 30,6 & 57,5 & 0,01530 & 0,05897 & 35,30 \\
\hline 5 & 60,5 & 10,7 & 0,35 & 3,04 & 0,31 & 0,0211 & 23,4 & 57,0 & 0,01506 & 0,05909 & 27,80 \\
\hline 6 & 68,4 & 7,3 & 0,39 & 2,95 & 0,22 & 0,0121 & 25,3 & 64,2 & 0,01545 & 0,05890 & 27,60 \\
\hline
\end{tabular}

En dicha tabla son presentados datos de humedad de equilibrio $U_{e}($ en base seca $)=M_{A e} / M_{S}$, siendo $M_{A e}$ masa de agua en un trozo de fruta en estado de equilibrio, $M_{S}$ masa de sólidos, dimensiones iniciales de una banana $L_{10}$ y $L_{20}$, y tiempo de secado $t$, para experimentos de Queiroz (1994); excepto $L_{2}$, que fue evaluada por Lima (1999). Los subíndices (0), (f) y (e) son, respectivamente, datos en el inicio, final y en condición de equilibrio, es decir, a $\mathrm{t} \rightarrow \infty$; UR es la humedad relativa del aire y $\mathrm{v}(\mathrm{m} / \mathrm{s})$ su velocidad.

Se resuelve un ejemplo con el ensayo (4) de la Tabla 1, para ilustrar cómo usar la metodología propuesta. Los resultados obtenidos para todos los ensayos de la Tabla 1 son comparados con los de Lima (1999), empleando un método numérico, en el cual una banana es un sólido esferoidal, con disminución de $L_{1}$ y $L_{2}$ en el proceso. Además son comparados con los cálculos de Ferreira y Costa (2009), que desarrollaron un modelo analítico con disminución de $R$, usando una ecuación para evaluar $R$ versus $M_{A}$. Se emplean datos experimentales de Queiroz (1994), de $Y=\left(M_{A}-M_{A \infty}\right) /\left(M_{A 0}-M_{A \infty}\right)$ versus $t$, y de ellos se obtiene $M_{A}=M_{A \infty}+\left(M_{A 0}-M_{A \infty}\right) Y_{\text {Queiroz }} \cdot M_{A 0}$ y $M_{A_{\infty}}$ fueron calculadas por Ferreira y Costa (2009), de datos de Queiroz (1994). Ferreira y Costa (2009) partieron de ecuaciones de $Y$ versus $t$, con tres términos, correlacionadas por Lima (1999). Acorde al procedimiento propuesto de evaluación de $\mathrm{Bi}_{R}$, $D_{\text {ef, }} M_{A 0}, M_{A \infty}, R_{m}, B i_{\llcorner}$y $k_{m}$, para el ensayo (4) de la Tabla 1, se debe:

i) Obtener $M_{A}$ versus t de datos de Queiroz (1994), de $Y=\left(M_{A}-M_{A \infty}\right) /\left(M_{A 0}-M_{A \infty}\right)$ versus t, reordenando la ecuación anterior, obteniendo la ecuación a continuación, con $\mathrm{M}_{\mathrm{A} 0}$ y $\mathrm{M}_{\mathrm{A}_{\infty}}$ calculados por Ferreira y Costa (2009), de datos de Queiroz (1994). Los datos de $Y, t, M_{A 0}, M_{A \infty}$, así como $M_{A}$ calculados con la Ec.(22), de $t_{0}=0 h$ a $t_{f}=35,3 h$, están presentados en la Tabla 2.

$$
M_{A}=0,61 g+(42,36 g-0,61 g) \cdot\{Y\}_{\text {Queiroz }}
$$

ii) Usar $\mathrm{M}_{\mathrm{A}}(\mathrm{g})$ y $\mathrm{t}(\mathrm{h})$ de las columnas (2) y (3) de la Tabla 2, correlacionando $\mathrm{x}=\mathrm{t}$ versus $\mathrm{y}=\mathrm{M}_{\mathrm{A}}$ de la Ec.(12), empleando, por ejemplo, un programa de regresión no lineal tal como el Lab Fit (Silva y Silva, 2003), manteniendo como parámetros libres $B i_{R}, D_{e f}, M_{A 0}, M_{A \infty}$ y $R_{m}$, resultando en:

$$
M_{A}=M_{A \infty}+\left(M_{A 0}-M_{A \infty}\right) \frac{4\left(1,03+B i_{R}\right)^{4}}{\left(2,4048 B_{R}\right)^{2}\left\{2,4048^{2}+\left(1,03+B i_{R}\right)^{2}\right\}} \exp \left\{-\left(\frac{2,4048 B i_{R}}{1,03+B i_{R}}\right)^{2} \frac{D_{e f} t}{R_{m}^{2}}\right\}
$$

Los parámetros de regresión de la Ec.(23), que están presentados en la Tabla 3 , son $A=B_{R}=3,182$, del cual se obtiene $\mu_{1}=1,817$ de la Ec.(11) y $B_{1}=0,914, B=D_{\text {ef }}=0,2676.10^{-5} \mathrm{~m}^{2} / \mathrm{h}=7,43.10^{-10} \mathrm{~m}^{2} / \mathrm{s}, C=R_{\mathrm{m}}$ $=0,01055 \mathrm{~m}, \mathrm{D}=\mathrm{M}_{\mathrm{A}^{\infty}}=1,178 \mathrm{~g}, \mathrm{E}=\mathrm{M}_{\mathrm{A} 0}=47,05 \mathrm{~g}$, que sustituidos en la $\mathrm{Ec}$.(23), da:

$$
M_{A}=1,178 g+(45,872 g) 0,914 \exp \left\{(1,817)^{2}\left(0,2676.10^{-5}\right) t /\left(0,1055.10^{-1}\right)^{2}\right\}
$$

Las Ecs.(22) y (24) son presentadas en la Tabla 4, así como las ecuaciones para los otros ensayos. 
iii) El radio promedio del ensayo 4 del presente trabajo, $R_{m}=0,01055 \mathrm{~m}$ difiere en $-2,7 \%$ de $R_{m}=$ 0,01084 m calculado al tiempo promedio $t_{m}=(35,3 \mathrm{~h}) / 2=17,65 \mathrm{~h}$, por Ferreira y Costa (2009), con una ecuación para $R$ versus $M_{A}$. La difusividad $D_{\text {ef }}=0,2676.10^{-5} \mathrm{~m}^{2} / \mathrm{h}=7,43.10^{-10} \mathrm{~m}^{2} / \mathrm{s}$ difiere en $2,5 \%$ de la $\mathrm{D}_{\mathrm{ef}}=7,25.10^{-10} \mathrm{~m}^{2} / \mathrm{s}$ de Lima (1999), del ensayo (4) de la Tabla 5 y modelo (III). El radio $\mathrm{R}_{\mathrm{m}}=0,01055 \mathrm{~m}$, el Bi en el radio de un cilindro infinito $B_{R}=3,182$ y $D_{\text {ef }}=7,43.10^{-10} \mathrm{~m}^{2} / \mathrm{s}$, son empleados en los cálculos a continuación, para obtener $\mathrm{Bi}_{L}$ y $\mathrm{k}_{\mathrm{m}}$, en el inicio y final del secado, de un trozo de banana con forma de un sólido esferoidal.

iv) Evaluar la longitud focal en el inicio del secado $L_{0}$, con datos del ensayo 4 de la Tabla 1 , para $L_{10} \approx R_{0}$ $=0,01530 \mathrm{~m} \mathrm{y}_{20}=0,05897 \mathrm{~m}$, el semieje menor y mayor, respectivamente, con la Ec.(13):

$$
L_{0}=\sqrt{\left(L_{20}^{2}-L_{10}^{2}\right)}=\sqrt{(0,05897 m)^{2}-(0,01530 m)^{2}}=0,05695 m
$$

v) Calcular el semieje mayor, de la etapa final del proceso $L_{2 f}$, de la Ec.(14), con $L_{0}$ de la Ec.(25), el radio promedio $L_{1 f} \approx R_{m}=0,01055 m$ y $L_{10} \approx R_{0}=0,01530 \mathrm{~m}$, del ensayo 4 de la Tabla 1 :

$$
L_{2 f}=L_{0} R_{m} / R_{0}=L_{0} L_{1 f} / L_{10}=(0,05695 \mathrm{~m}) 0,01055 \mathrm{~m} / 0,01530 \mathrm{~m}=0,03927 \mathrm{~m}
$$

vi) Evaluar la longitud focal $L_{f}$ del final del secado, con la Ec.(15), con datos de $L_{1 f} \approx R_{m}=0,01055 \mathrm{~m}$ y $L_{2 f}=0,03927 \mathrm{~m}$ de la Ec.(26), resultando en:

$$
L_{f}=\sqrt{\left(L_{2 f}^{2}-L_{1 f}^{2}\right)}=\sqrt{\left(L_{2 f}^{2}-L_{m}^{2}\right)}=\sqrt{(0,03927 m)^{2}-(0,01055 m)^{2}}=0,03783 m
$$

vii) Calcular el $\mathrm{Bi}$ de un sólido esferoidal en el inicio y final del proceso $\mathrm{Bi}_{\mathrm{LO}}$ y $\mathrm{Bi}_{\mathrm{Lf}}$, respectivamente, con las Ecs.(16) y (17), $L_{0}$ y $L_{f}$ de las Ecs.(25) y (27), respectivamente, $B_{R}=3,182$ y $R_{m}=0,01055 \mathrm{~m}$ :

$$
\mathrm{Bi}_{\mathrm{L} 0}=\mathrm{Bi}_{\mathrm{R}} \mathrm{L}_{0} / \mathrm{R}_{\mathrm{m}}=(3,182) 0,05695 \mathrm{~m} / 0,01055 \mathrm{~m}=17,18
$$

$$
B i_{L f}=B i_{R} L_{f} / R_{m}=(3,182) 0,03783 m / 0,01055 m=11,41
$$

Los números de Biot $\mathrm{Bi}_{\mathrm{L} 0}=17,18$ y $\mathrm{Bi}_{\mathrm{Lf}}=11,41$ de las Ecs.(28) y (29), difieren, respectivamente, en $-2 \%$ de $B \mathrm{i}_{\mathrm{Lf}}=17,52$ y $2,4 \%$ de $\mathrm{Bi}_{\mathrm{Lf}}=11,14$, del ensayo (4) de la Tabla 5 y modelo (III) de Lima.

viii) Calcular el coeficiente externo de transferencia de materia para el sólido esferoidal, en el inicio $\mathrm{k}_{\mathrm{L} 0} \mathrm{y}$ final del secado $\mathrm{k}_{\mathrm{Lf}}$, respectivamente, con las Ecs.(18) y (19), Bi $\mathrm{B}_{\mathrm{LO}}$ y $\mathrm{Bi}_{\mathrm{Lf}}$, de las Ecs.(28) y (29), respectivamente, $L_{0}$ y $L_{f}$ de las Ecs.(25) y (27), respectivamente, y $D_{\text {ef }}=7,43.10^{-10} \mathrm{~m}^{2} / \mathrm{s}$ :

$$
\begin{aligned}
& \mathrm{k}_{\mathrm{L} 0}=\mathrm{Bi}_{\mathrm{L} 0} \mathrm{D}_{\text {ef }} / \mathrm{L}_{0} \cong 17,18\left(7,43 \cdot 10^{-10} \mathrm{~m}^{2} / \mathrm{s}\right) 0,5695 \mathrm{~m} \cong 22,42 \cdot 10^{-8} \mathrm{~m} / \mathrm{s} \\
& \mathrm{k}_{\mathrm{Lf}}=\mathrm{Bi}_{\mathrm{Lf}} \mathrm{D}_{\text {ef }} / \mathrm{L}_{\mathrm{f}} \cong 11,41\left(7,43 \cdot 10^{-10} \mathrm{~m}^{2} / \mathrm{s}\right) / 0,03783 \mathrm{~m} \cong 22,42 \cdot 10^{-8} \mathrm{~m} / \mathrm{s}
\end{aligned}
$$

Los coeficientes $k_{\mathrm{L} 0}$ y $\mathrm{k}_{\mathrm{Lf}}$ difieren en $0,5 \%$ de los $\mathrm{k}_{\mathrm{L} 0}=\mathrm{k}_{\mathrm{Lf}}=22,3 \cdot 10^{-8} \mathrm{~m} / \mathrm{s}$ de Lima, del ensayo (4) de la Tabla 5. En la Tabla 3 se presentan los principales resultados del actual artículo para los ensayos (1) al (6); de igual manera a la desarrollada para el ensayo (4). Son usados datos de $Y$ versus t así como otros datos de Queiroz (1994), para obtener ecuaciones similares a la Ec.(22); siendo presentadas ecuaciones equivalentes a las Ecs.(22) y (24) en la Tabla 4. Las ecuaciones similares a la Ec.(22), con auxilio de la Ec.(23), son usadas para evaluar parámetros y obtener ecuaciones equivalentes a la Ec.(24).

Tabla 2: Masa de agua versus tempo, para un trozo de banana del ensayo (4). 


\begin{tabular}{|c|c|c|c|}
\hline$Y=\left(\frac{M_{A}-M_{A \infty}}{M_{A 0}-M_{A \infty}}\right)_{\text {Queiroz }}$ & $t(h)_{\text {Queiroz }}$ & $\begin{array}{c}\mathrm{M}_{\mathrm{A}}(\mathrm{g})= \\
=0,61 \mathrm{~g}+(42,36 \mathrm{~g}-0,61 \mathrm{~g}) \cdot\{\mathrm{Y}\}_{\text {Queiroz }} \\
\text { Ec. }(22)\end{array}$ & $\begin{array}{c}M_{A}(g)= \\
=1,18 g+(41,93 g) \exp (-0,0794 \\
\text { Ec. }(24)\end{array}$ \\
\hline 1,0000 & 0,0000 & 42,36 & 42,57 \\
\hline 0,9315 & 1,0000 & 39,50 & 39,38 \\
\hline 0,8599 & 2,0000 & 36,51 & 36,44 \\
\hline 0,7937 & 3,0000 & 33,75 & 33,72 \\
\hline 0,7322 & 4,0167 & 31,18 & 31,17 \\
\hline 0,6764 & 5,0167 & 28,85 & 28,86 \\
\hline 0,6250 & 6,0167 & 26,70 & 26,73 \\
\hline 0,5783 & 7,0167 & 24,75 & 24,76 \\
\hline 0,5351 & 8,0167 & 22,95 & 22,94 \\
\hline 0,4951 & 9,0167 & 21,28 & 21,27 \\
\hline 0,4577 & 10,0167 & 19,72 & 19,72 \\
\hline 0,4232 & 11,0167 & 18,28 & 18,29 \\
\hline 0,3914 & 12,0167 & 16,95 & 16,97 \\
\hline 0,3621 & 13,0167 & 15,73 & 15,76 \\
\hline 0,3350 & 14,0167 & 14,60 & 14,63 \\
\hline 0,3102 & 15,0167 & 13,56 & 13,60 \\
\hline 0,2871 & 16,0167 & 12,60 & 12,64 \\
\hline 0,2659 & 17,0167 & 11,71 & 11,76 \\
\hline 0,2462 & 18,0167 & 10,89 & 10,94 \\
\hline 0,2280 & 19,0167 & 10,13 & 10,19 \\
\hline 0,2115 & 20,0167 & 9,44 & 9,49 \\
\hline 0,1968 & 21,0167 & 8,83 & 8,85 \\
\hline 0,1829 & 22,0167 & 8,25 & 8,26 \\
\hline 0,1699 & 23,0167 & 7,70 & 7,72 \\
\hline 0,1580 & 24,0167 & 7,21 & 7,21 \\
\hline 0,1470 & 25,0167 & 6,75 & 6,75 \\
\hline 0,1368 & 26,0167 & 6,32 & 6,32 \\
\hline 0,1275 & 27,0167 & 5,93 & 5,92 \\
\hline 0,1188 & 28,0167 & 5,57 & 5,56 \\
\hline 0,1109 & 29,0167 & 5,24 & 5,22 \\
\hline 0,1036 & 30,0167 & 4,94 & 4,91 \\
\hline 0,0965 & 31,0167 & 4,64 & 4,62 \\
\hline 0,0899 & 32,0167 & 4,36 & 4,36 \\
\hline 0,0837 & 33,0167 & 4,10 & 4,11 \\
\hline 0,0780 & 34,0167 & 3,87 & 3,88 \\
\hline 0,0729 & 35,0167 & 3,65 & 3,68 \\
\hline 0,0719 & 35,2667 & 3,61 & 3,63 \\
\hline
\end{tabular}

Comparando las $D_{\text {ef }}$ de la Tabla 5, calculadas por Lima (1999) con el modelo (III) y las del actual trabajo, resulta en una desviación promedio $X_{\text {prom }}=-1,4 \%$ y estándar $\sigma_{n-1}=2,4 \%$. Para los coeficientes de transferencia de materia de un sólido esferoidal $k_{L}$, se obtiene $X_{\text {prom }}=-3,2 \%$ y $\sigma_{n-1}=3,6 \%$, y para los $B i$ de un sólido esferoidal $B i_{L}$, resulta en $X_{\text {prom }}=1,3$ y $\sigma_{n-1}=4,3 \%$. Comparando $D_{\text {ef, }}, k_{L}$ y $B i_{L}$ de $\operatorname{Lima}(1999)$ con el modelo (III) y los de Ferreira y Costa (2009), resultan, respectivamente, en $X_{\text {prom }}=-6,1 \%$ y $\sigma_{n-1}=$ $10,3 \%, X_{\text {prom }}=-10,8 \%$ y $\sigma_{n-1}=4,3 \%$, y $X_{\text {prom }}=-4,0 \%$ y $\sigma_{n-1}=10,4 \%$. Es mejor el ajuste de parámetros del presente trabajo en relación al de Ferreira y Costa (2009), usando como referencia los cálculos numéricos de Lima (1999). En el actual artículo se emplean menos parámetros experimentales que en el de Ferreira y Costa (2009) o de Lima (1999). Tampoco se necesitan de correlaciones para evaluar parámetros de transferencia de materia en la presente investigación ni en el de Ferreira y Costa (2009). 
Tabla 3: Parámetros evaluados con el modelo propuesto para los ensayos E (1) al (6).

\begin{tabular}{|c|c|c|c|c|c|c|c|c|c|c|c|}
\hline $\mathrm{E}$ & $\begin{array}{c}\mathrm{Bi}_{\mathrm{R}} \\
(\operatorname{adim} .)\end{array}$ & $\begin{array}{c}\mathrm{D}_{\mathrm{ef} .} \cdot 10^{10} \\
\left(\mathrm{~m}^{2} / \mathrm{s}\right)\end{array}$ & $\begin{array}{c}\mathrm{R}_{\mathrm{m}} \\
(\mathrm{m})\end{array}$ & $\begin{array}{c}\mathrm{M}_{\mathrm{A} \infty} \\
(\mathrm{g})\end{array}$ & $\begin{array}{c}\mathrm{M}_{\mathrm{A} 0} \\
(\mathrm{~g})\end{array}$ & $\begin{array}{c}\mathrm{L}_{0} \\
(\mathrm{~m})\end{array}$ & $\begin{array}{c}\mathrm{L}_{2 \mathrm{f}} \\
(\mathrm{m})\end{array}$ & $\begin{array}{c}\mathrm{L}_{\mathrm{f}} \\
(\mathrm{m})\end{array}$ & $\begin{array}{c}\mathrm{Bi}_{\mathrm{LO}} \\
(\operatorname{adim} .)\end{array}$ & $\begin{array}{c}\mathrm{Bi}_{\mathrm{Lf}_{\mathrm{f}}} \\
(\operatorname{adim} .)\end{array}$ & $\begin{array}{c}\mathrm{k}_{\mathrm{L}} \cdot 10^{8} \\
(\mathrm{~m} / \mathrm{s})\end{array}$ \\
\hline 1 & 6,12 & 1,57 & 0,01052 & 1,243 & 55,07 & 0,05629 & 0,03672 & 0,03518 & 32,73 & 20,45 & 9,15 \\
\hline 2 & 6,35 & 2,46 & 0,01007 & 2,431 & 51,56 & 0,05665 & 0,03636 & 0,03494 & 35,71 & 22,02 & 15,53 \\
\hline 3 & 4,59 & 4,52 & 0,01006 & 1,339 & 47,96 & 0,05701 & 0,03768 & 0,03632 & 26,00 & 16,56 & 20,62 \\
\hline 4 & 3,18 & 7,43 & 0,01055 & 1,178 & 47,05 & 0,05695 & 0,03927 & 0,03783 & 17,18 & 11,41 & 22,42 \\
\hline 5 & 3,59 & 7,10 & 0,01004 & 0,941 & 45,77 & 0,05714 & 0,03809 & 0,03675 & 20,43 & 13,14 & 25,38 \\
\hline 6 & 3,30 & 8,48 & 0,01098 & $-1,076$ & 48,23 & 0,05684 & 0,04039 & 0,03887 & 17,06 & 11,67 & 25,45 \\
\hline
\end{tabular}

Tabla 4: Ecuaciones auxiliares de cálculo

\begin{tabular}{|c|c|c|}
\hline Ensayo & $\begin{array}{l}\text { Ecuación obtenida de datos de Queiroz } \\
\text { (1994) }\end{array}$ & Ecuación del presente trabajo \\
\hline 1 & $M_{A}=2,02 g+(48,43 g-2,02 g) \cdot\{Y\}_{\text {Queiroz }}$ & $M_{A}=1,24 g+(53,83 g) 0,848 \exp \left\{-(2,058)^{2} \frac{0,5666 \cdot 10^{-6} t}{(0,01052)^{2}}\right\}$ \\
\hline 1 & $M_{A}=0,95 g+(45,16 g-0,95 g) \cdot\{Y\}_{\text {Queiroz }}$ & $M_{A}=2,43 g+(49,13 g) 0,845 \exp \left\{-(2,069)^{2} \frac{0,8868 \cdot 10^{-6} t}{(0,01007)^{2}}\right\}$ \\
\hline 3 & $M_{A}=0,77 g+(42,79 g-0,77 g) \cdot\{Y\}_{\text {Queiroz }}$ & $M_{A}=1,34 g+(46,62 g) 0,877 \exp \left\{-(1,964)^{2} \frac{0,1628.10^{-5} t}{(0,01006)^{2}}\right\}$ \\
\hline 4 & $M_{A}=0,61 g+(42,36 g-0,61 g) \cdot\{Y\}_{\text {Queiroz }}$ & $M_{A}=1,18 g+(45,87 g) 0,914 \exp \left\{-(1,817)^{2} \frac{0,2676.10^{-5} t}{(0,01055)^{2}}\right\}$ \\
\hline 5 & $M_{A}=0,29 g+(41,40 g-0,29 g) \cdot\{Y\}_{\text {Queiroz }}$ & $M_{A}=0,94 g+(44,83 g) 0,901 \exp \left\{-(1,869)^{2} \frac{0,2556 \cdot 10^{-5} t}{(0,01004)^{2}}\right\}$ \\
\hline 6 & $M_{A}=0,18 g+(43,11 g-0,18 g) \cdot\{Y\}_{\text {Queiroz }}$ & $M_{A}=-1,08 g+(49,31 g) 0,910 \exp \left\{-(1,832)^{2} \frac{0,3053 \cdot 10^{-5} t}{(0,01098)^{2}}\right\}$ \\
\hline
\end{tabular}

Tabla 5: Parámetros obtenidos por Lima (1999), Ferreira y Costa (2009) y en la actual investigación.

\begin{tabular}{|c|c|c|c|c|c|c|c|c|c|c|}
\hline \multicolumn{5}{|c|}{$\begin{array}{l}\text { Modelo III de sólido esferoidal (Lima, } \\
\text { 1999). Se emplea condición de contorno } \\
\text { convectiva + disminución de volumen. }\end{array}$} & \multicolumn{3}{|c|}{$\begin{array}{l}\text { Modelo del presente artículo. } \\
\text { No se necesitan de datos de } \\
\text { disminución del radio. }\end{array}$} & \multicolumn{3}{|c|}{$\begin{array}{l}\text { Modelo de cilindro infinito (Ferreira } \\
\text { y Costa, 2009). Se emplean datos } \\
\text { de disminución del radio. }\end{array}$} \\
\hline $\begin{array}{l}\mathrm{E} \\
\mathrm{n} \\
\mathrm{s} \\
\mathrm{a} \\
\mathrm{y} \\
\mathrm{o}\end{array}$ & $\begin{array}{l}\mathrm{T}_{\mathrm{a}} \\
\left({ }^{\circ} \mathrm{C}\right)\end{array}$ & $\begin{array}{c}D_{\text {ef }} \cdot 10^{10} \\
\left(\mathrm{~m}^{2} / \mathrm{s}\right)\end{array}$ & $\begin{array}{r}k_{m} \cdot 10^{8} \\
(\mathrm{~m} / \mathrm{s})\end{array}$ & $\begin{array}{c}\mathrm{Bi}_{\mathrm{L}} \\
\text { (adim.) }\end{array}$ & $\begin{array}{l}D_{\text {ef }} \cdot 10^{10} \\
\left(\mathrm{~m}^{2} / \mathrm{s}\right)\end{array}$ & $\begin{array}{r}k_{m} \cdot 10^{8} \\
(\mathrm{~m} / \mathrm{s})\end{array}$ & $\begin{array}{c}\mathrm{Bi}_{\mathrm{L}} \\
\text { (adim.) }\end{array}$ & $\begin{array}{l}D_{e f} \cdot 10^{10} \\
\left(\mathrm{~m}^{2} / \mathrm{s}\right)\end{array}$ & $\begin{array}{r}k_{m} \cdot 10^{8} \\
(\mathrm{~m} / \mathrm{s})\end{array}$ & $\begin{array}{c}\mathrm{Bi}_{\mathrm{L}} \\
\text { (adim.) }\end{array}$ \\
\hline 1 & 29,9 & 1,65 & 10,10 & $\begin{array}{l}34,46 \\
18,40\end{array}$ & 1,57 & $\begin{array}{l}9,15 \\
9,15\end{array}$ & $\begin{array}{l}32,73 \\
20,45\end{array}$ & 1,35 & $\begin{array}{l}8,80 \\
8,80\end{array}$ & $\begin{array}{l}36,72 \\
19,61\end{array}$ \\
\hline 2 & 39,9 & 2,48 & 15,53 & $\begin{array}{l}35,47 \\
21,38\end{array}$ & 2,46 & $\begin{array}{l}15,53 \\
15,53\end{array}$ & $\begin{array}{l}35,71 \\
22,02\end{array}$ & 2,32 & $\begin{array}{l}12,86 \\
12,86\end{array}$ & $\begin{array}{l}31,38 \\
18,91\end{array}$ \\
\hline 3 & 49,9 & 4,57 & 21,35 & $\begin{array}{l}26,64 \\
15,75\end{array}$ & 4,52 & $\begin{array}{l}20,62 \\
20,62\end{array}$ & $\begin{array}{l}26,00 \\
16,56\end{array}$ & 4,01 & $\begin{array}{l}19,17 \\
19,17\end{array}$ & $\begin{array}{l}27,29 \\
16,14\end{array}$ \\
\hline 4 & 60,2 & 7,25 & 22,30 & $\begin{array}{l}17,52 \\
11,14\end{array}$ & 7,43 & $\begin{array}{l}22,42 \\
22,42\end{array}$ & $\begin{array}{l}17,18 \\
11,41\end{array}$ & 6,95 & $\begin{array}{l}21,16 \\
21,16\end{array}$ & $\begin{array}{l}17,35 \\
11,03\end{array}$ \\
\hline 5 & 60,5 & 7,30 & 26,15 & $\begin{array}{l}20,47 \\
12,89\end{array}$ & 7,10 & $\begin{array}{l}25,38 \\
25,38\end{array}$ & $\begin{array}{l}20,43 \\
13,14\end{array}$ & 6,71 & $\begin{array}{l}24,46 \\
24,46\end{array}$ & $\begin{array}{l}20,84 \\
13,12\end{array}$ \\
\hline 6 & 68,4 & 8,63 & 26,56 & $\begin{array}{l}17,49 \\
11,23\end{array}$ & 8,48 & $\begin{array}{l}25,45 \\
25,45\end{array}$ & $\begin{array}{l}17,06 \\
11,67\end{array}$ & 9,69 & $\begin{array}{l}23,15 \\
23,15\end{array}$ & $\begin{array}{c}13,57 \\
8,71\end{array}$ \\
\hline
\end{tabular}


Los números de Biot para cilindro infinito evaluados en éste trabajo están en el rango $3,18 \leq \mathrm{Bi}_{\mathrm{R}} \leq 6,35$, acorde a los resultados presentados en la Tabla 3; siendo adecuado emplear la Ec.(11) para calcular $\mu_{1}$, pues todos $\mathrm{Bi}_{\mathrm{R}}>2$. Todos ésos $\mathrm{Bi}$ están dentro del rango de $\mathrm{Bi}$ intermedio, $0 \leq \mathrm{Bi} \leq 100$, es decir, en el cual hay resistencia interna y externa a la transferencia de materia, acorde a los criterios propuestos en el libro de Luikov (1968). El rango de Bi de sólido esferoidal de la Tabla 3 es $11,41 \leq \mathrm{Bi}_{\mathrm{L}} \leq 35,71$.

Cuando se calcula el valor del semieje mayor del final del proceso $L_{2 f}$, de la $E c$.(14), es decir, a partir de $L_{2 f} \approx\left(L_{0} / R_{0}\right) R_{m}$ y $L_{f}$ por la Ec.(15), está implícito que ocurre cambio de $R$, así como de las dimensiones $L_{1}$, $L_{2}$ y $L$ en el secado. No es necesario conocer datos de disminución de dimensiones de un trozo de banana, pero se supone que ocurre cambio de dichas dimensiones, acorde a las relaciones presentadas entre $L_{2 f}, L_{0}, L_{f}$ y $R$. El cambio en $R$ es obtenido de la correlación de datos de $M_{A}$ versus $t$, de la Ec.(12) o (24), resultando en un radio promedio $R_{m}$, como el presentado en la Ec.(23) o (24). Se puede evaluar el radio $R$ versus $M_{A}$ de un trozo de fruta, con la metodología del presente artículo. A partir de una ecuación del tipo $R=a+b M_{A}$, se calculan (a) y (b), haciendo $a t=0 h, R=R_{0}$ y $M_{A}=M_{A 0}$, y a tiempo promedio $t=$ $t_{m}$, que para el ensayo (4) es $t_{m}=(35,3 / 2) h=17,65 \mathrm{~h}$ de la Tabla $1, R=R_{m}$ y $M_{A}=M_{A}\left(t_{m}\right)$; siendo el $R_{m}$ obtenido de la Ec.(12) o (23) y $M_{A}\left(t_{m}\right)$ a $t=t_{m}$ de la Ec.(24). Los valores de radio $R$ calculados con el procedimiento descrito son similares a los evaluados por Ferreira y Costa (2009).

Facilita la evaluación de los parámetros con un programa de regresión, $A=B i_{R}, B=D_{\text {ef }}, C=R_{m}, D=M_{A_{\infty}}$ y $E=M_{A 0}$, de la Ec.(12), y el cálculo de $B_{L} y_{k_{m}}$, si se conoce el rango de cada uno de ellos o se dispone de valores obtenidos por otros investigadores. Por ejemplo, $D_{\text {ef }}$ está aproximadamente en el rango $10^{-10}<$ $D_{\text {ef }}\left(\mathrm{m}^{2} / \mathrm{s}\right)<10^{-9} \mathrm{y}_{\mathrm{m}}$ en el rango $10^{-8}<\mathrm{k}_{\mathrm{m}}(\mathrm{m} / \mathrm{s})<10^{-7}$, para los ensayos (1) al (6), acorde a los cálculos de Lima (1999). $R_{m}$ puede ser evaluado con la metodología del párrafo anterior, $E=M_{A 0}$ del contenido acuoso de una banana, que es de aproximadamente $75 \%$, y para $D=M_{A_{\infty}}$ puede ser asignado un valor mucho menor que $M_{A 0}$. Cuando se dispone de datos experimentales de $M_{A}$ versus $t$, otra opción de cálculo de los parámetros citados, es minimizar el cuadrado de las desviaciones de $\mathrm{M}_{\mathrm{A}}$ versus t, así como el promedio de sus desviaciones; como fue realizado en el presente trabajo.

\section{CONCLUSIONES}

Los parámetros $D_{\text {ef }}, B_{\llcorner}$y $k_{m}$ evaluados con la metodología del presente trabajo, se ajustan mejor a los calculados por Lima (1999) en su modelo (III), usando un método numérico, que los resultados de Ferreira y Costa (2009). Los parámetros $B_{L}, B i_{R}, D_{e f}, k_{m}, M_{A 0}$ y $M_{A \infty}$ son obtenidos naturalmente del método desarrollado, sin necesidad de experimentos por separado para calcularlos ni de correlaciones para evaluarlos y tampoco de datos de disminución de las dimensiones de un trozo de banana en su secado.

La disminución de volumen puede ser admitida en modelos de secado de frutas, pues resultan en $\mathrm{Bi}_{\mathrm{L}}, \mathrm{Bi}_{\mathrm{R}}$, $D_{\text {ef }} y k_{m}$ que, a veces, representan mejor el fenómeno, que en modelados de volumen constante (Ferreira y Costa, 2009; Lima, 1999). Asimismo es posible evaluar parámetros de secado de frutas sin que esté explícita su disminución de volumen y sin conocer datos de tal disminución de dimensiones, pero que representen bien el fenómeno, como se demostró con la metodología de la presente investigación.

\section{REFERENCIAS}

Abalone, R., A. Gastón y M.A. Lara; Simulación Numérica del Proceso de Secado de un Material Anisotrópico, Engenharia Térmica: 1, 47-55 (2001).

Bird, R.B., W.E. Stewart y E.N. Lightfoot; Transport Phenomena, John Wiley, New York, USA (2002).

Costa, A.R.S.; Sistema de Secado Solar para Frutos Tropicales y Modelado del Secado de Banana en un Secador de Columna Estática, Tesis doctoral, UFRN - Universidad Federal de Río Grande del Norte, Natal, Brasil (2008). (En portugués y disponible en www.ufrn.br.)

Crank, J.; The Mathematics of Diffusion, Clarendon Press, Oxford, England (1976).

Ferreira, S.R. y A.R.S. Costa; Parámetros de Transferencia de Materia en el Secado de Frutas, Información Tecnológica: 20(2), 89-104 (2009). 
Karim, Md.A. y M.N.A. Hawlader; Drying Characteristics of Banana: Theoretical Modelling and Experimental Validation, Journal of Food Engineering: 70, 35-45 (2005a).

Karim, Md.A. y M.N.A. Hawlader; Mathematical Modelling and Experimental Investigation of Tropical Fruits Drying, International Journal of Heat and Mass Transfer: 48, 4914-4925 (2005b).

Kaya, A., O. Aydın y I. Dincer; Experimental and Numerical Investigation of Heat and Mass Transfer During Drying of Hayward Kiwi Fruits (Actinidia Deliciosa Planch), Journal of Food Engineering: 88, 323330 (2008).

Lima, A.G.B., M.R. Queiroz y S.A. Nebra; Simultaneous Moisture Transport and Shrinkage During Drying of Solids with Ellipsoidal Configuration, Chemical Engineering Journal: 86, 85-93 (2002).

Lima, A.G.B.; Fenómeno de Difusión en Sólidos Esferoidales Prolatos. Estudio de Caso: Secado de Banana, Tesis doctoral, UNICamp - Universidad Estadual de Campinas, Facultad de Ingeniería Mecánica, Campinas, Brasil (1999). (En portugués y disponible en http://libdigi.unicamp.br/; el código del trabajo es vtls000188465.)

Luikov, A.V.; Analytical Heat Diffusion Theory, Academic Press, New York, USA (1968).

Luikov, A.V.; Heat and Mass Transfer in Capillary Porous Bodies, Pergamon Press, New York, USA (1966).

Mabrouk, S.B., B. Khiari y M. Sassi; Modelling of Heat and Mass Transfer in a Tunnel Dryer, Applied Thermal Engineering: 26, 2110-2118 (2006).

Mariani, V.C., A.G.B. Lima y L.S. Coelho; Apparent Thermal Diffusivity Estimation of the Banana During Drying Using Inverse Method, Journal of Food Engineering: 85, 569-579 (2008).

Pandey, R.N., S.K. Srivastava y M.D. Mikhailov; Solutions of Luikov Equations of Heat and Mass Transfer in Capillary Porous Bodies Through Matrix Calculus: a New Approach, International Journal of Heat and Mass Transfer: 42, 2649-2660 (1999).

Queiroz, M.R.; Estudio Teórico-experimental de la Cinética de Secado de Bananas, Tesis doctoral, UNICamp - Universidad Estadual de Campinas, Facultad de Ingeniería Mecánica (1994). (En portugués y disponible en http://libdigi.unicamp.br/; el código del trabajo es vtls000082363.)

Schwartzberg, H. G.; Mathematical Analysis of the Freezing and Thawing of Foods, AIChE Summer National Meeting, Detroit, Michigan (1981).

Silva, W.P. y C.M.D.P.S., Silva; LAB Fit Ajuste de Curvas (Regresión no Lineal y Tratamiento de Datos) v.7.2.14c (2003). (En portugués y disponible en http://www.angelfire.com/rnb/labfit/index_p.htm.)

Srikiatden, J. y J.S. Roberts; Predicting Moisture Profiles in Potato and Carrot During Convective Hot Air Drying Using Isothermally Measured Effective Diffusivity, Journal of Food Engineering: 84, 516-525 (2008).

Teruel, B., L.A. Cortez, P. Leal y A.G.B Lima; Estudio Teórico del Enfriamiento de Frutas de Distintas Geometrías con Circulación Forzada de Aire, Ciência e Tecnologia de Alimentos: 21(2), 228-235, mayoagosto (2001). (En portugués.)

Wu, Y. y J. Irudayaraj; Analysis of Heat, Mass and Pressure Transfer in Starch Based Food Systems, Journal of Food Engineering: 29, 399-414 (1996). 